\title{
Clinical experience with CorMatrix extracellular matrix in the surgical treatment of mitral valve disease
}

\author{
Marc W. Gerdisch, MD, FACS, FACC, Richard J. Shea, MD, and Michael D. Barron, MD
}

\begin{abstract}
Objectives: To determine the clinical utility of an acellular bioscaffold as a structural material for mitral valve repair $(\mathrm{MVr})$.
\end{abstract}

\begin{abstract}
Methods: This was a retrospective study of patients undergoing partial or subtotal leaflet replacement and/or leaflet extension to treat mitral regurgitation or acute endocarditis and/or reconstruction of atrial-ventricular continuity after annular decalcification. The material used for repair was a bioresorbable extracellular matrix (ECM) material indicated for cardiac tissue repair (CorMatrix Cardiovascular, Inc, Roswell, Ga). After the necessary debridement, the ECM bioscaffold was tailored and sewn to the native mitral valve tissue. Intraoperative photographs and serial, follow-up echocardiograms were used for evaluation.
\end{abstract}

\begin{abstract}
Results: From September 2008 to February 2013, MVr requiring the addition of patch material was performed in 19 patients. The median echocardiographic follow-up was 10.9 months (range, 4 days to 48 months). One early and 2 late deaths were unrelated to MVr. No perioperative or late strokes occurred. Two patients with a history of cancer, chemotherapy, and radiotherapy experienced failure of the initial MVr, necessitating reintervention. The other MVrs continued to show good valvular function and no calcification on echocardiographic follow-up of 4 days to 48 months.
\end{abstract}

Conclusions: The ECM bioscaffold is a satisfactory material for MVr in a variety of surgical situations, including endocarditis. It appears to resist calcification and infection. Additional studies are warranted to determine the long-term durability of repairs made with ECM, and its appropriate use in patients who have previously undergone radiotherapy or chemotherapy. (J Thorac Cardiovasc Surg 2014;148:1370-8)

Mitral valve (MV) repair (MVr) offers clinical advantages compared with MV replacement (MVR); therefore, evidence-based guidelines have recommended $\mathrm{MVr}$ instead of MVR when possible. ${ }^{1-4}$ For many repairs, leaflet augmentation or annular reconstruction with foreign material patching could be required.

A patch material sturdy enough to withstand the mechanical forces within the heart but that does not provoke a foreign body response would be desirable. The biosynthetic materials commonly used for cardiac tissue repair, such as xenografts (eg, glutaraldehyde bovine pericardium), and synthetic materials, such as polytetrafluoroethylene or polyethylene terephthalate, might not be

\footnotetext{
From the Cardiac Surgery Associates, Franciscan St Francis Heart Center, Indianapolis, Ind.

CorMatrix Cardiovascular provided funds for professional medical editing; the authors maintained control over the study and report.

Disclosures: Marc W. Gerdisch reports consulting and lecture fees from, equity ownership in, and is on the Advisory Board of CorMatrix Cardiovascular, Inc. He also reports consulting and lecture fees from and equity ownership in Atricure, and lecture fees from Medtronic. CorMatrix Cardiovascular provided funds for professional medical editing. The authors maintained control over the study and manuscript. All other authors have nothing to disclose with regard to commercial support.

Received for publication June 12, 2013; revisions received Oct 3, 2013; accepted for publication Oct 29, 2013; available ahead of print Dec 10, 2013.

Address for reprints: Marc W. Gerdisch, MD, FACS, FACC, Cardiac Surgery Associates, Franciscan St Francis Heart Center, 5255 E Stop 11 Rd, Suite 200, Indianapolis, IN 46237 (E-mail: mgerdisch@openheart.net).

0022-5223/\$36.00

Copyright (c) 2014 by The American Association for Thoracic Surgery

http://dx.doi.org/10.1016/j.jtcvs.2013.10.055
}

suitable for MVr, because their use results in the permanent implantation of an immunologically reactive foreign body, raising concerns about inflammation, calcification, and degeneration. ${ }^{1-7}$ Arguably the best-accepted material for valve repair has been autologous pericardium. $^{8-11}$ However, concerns about fresh pericardium's lack of material strength and long-term durability have made glutaraldehyde fixation common, potentially undermining its advantages as a natural biologic material. Finally, because synthetic and fixed biologic materials are inert, they are incapable of adapting to somatic growth in young patients.

During the past 4 years, we have used a material composed of the decellularized extracellular matrix (ECM) of porcine small-intestinal submucosa for $\mathrm{MVr} .{ }^{12}$ Unlike previously used materials, the ECM bioscaffold is a noncrosslinked, cell-free bioscaffold that serves as a biologically active substrate for constructive remodeling. It has been shown to recruit host stem cells in preclinical models of cardiac and other tissue types and has exhibited growth potential in a preclinical vascular graft model. ${ }^{12-17}$ These experimental studies have demonstrated that the ECM provides the unique environment necessary to promote progenitor cell attachment, migration, expansion, and maturation. With time, the ECM bioscaffold will be remodeled by the host progenitor cells to resemble native tissue.

Preliminary findings in both experimental animals and humans have shown that the ECM bioscaffold does not evoke a strong inflammatory response and is resistant to 

Abbreviations and Acronyms
$\mathrm{ECM}=$ extracellular matrix
$\mathrm{MV}=$ mitral valve
$\mathrm{MVr}=$ mitral valve repair
$\mathrm{MVR}=$ mitral valve replacement

calcification. ${ }^{12,18,19}$ It is available for clinical use in the United States and Europe, with indications for pericardial closure, cardiac tissue repair, and carotid artery repair.

\section{METHODS}

\section{Study Design and Patients}

The Western institutional review board approved the present retrospective chart review. Patient consent requirements were waived. Preoperative, intraoperative, and follow-up data were collected from the charts of all patients who had undergone MVr after August 2007, when the ECM biomaterial was adopted into our practice.

\section{Operative Techniques}

All procedures were performed at the same institution by the same surgeon (M.W.G.). Transesophageal echocardiography was used to confirm the valve pathologic features immediately before surgery and to evaluate the repair afterward. The surgical approaches and procedures varied according to the specific pathologic features; however, generally, a midline sternotomy was performed. Cardiopulmonary bypass was initiated and the heart arrested with cold antegrade and retrograde cardioplegia. Cardioplegia was subsequently administered intermittently. The left atrium was entered through the interatrial groove and the diseased MV tissue debrided or resected as necessary. The resulting leaflet defects, or other pre-existing deficiencies, were patched with the ECM bioscaffold (CorMatrix Cardiovascular, Inc, Roswell, Ga) tailored to the appropriate size and shape. The material was sutured into place with running 5-0 polypropylene suture (Prolene; Ethicon Inc, Somerville, NJ). Horizontal sutures of 2-0 polyester (Ethibond, Ethicon Inc) were placed around the circumference of the annulus using an appropriately sized annuloplasty ring (Physio, Edwards Lifesciences, Irvine, Calif; ATS, LeviBio Medica, Rome, Italy; CG Future, Medtronic, Minneapolis, Minn; or 3D, Medtronic). The potential for the development of systolic anterior motion and resulting obstruction of the left ventricular outflow tract was minimized using best-practice techniques, such as "sliding leaflet" valvuloplasty. ${ }^{20-22}$

For decalcification and reconstruction of the mitral annulus, the valve leaflets were dissected away from the annulus when possible to allow removal of calcified tissue and then reattached using 4-0 polypropylene suture. The resulting defects in annular tissue were repaired by sewing into place an appropriately tailored piece of the ECM bioscaffold using 4-0 polypropylene sutures. The patch was made redundant and then imbricated into the defect to fill it with ECM. An annuloplasty ring was sewn into place, as described.

The chordae tendineae were either preserved or replaced with polytetrafluoroethylene neochordae (Gore-Tex, W L Gore \& Assoc, Inc, Flagstaff, Ariz). Concurrent procedures (eg, valve replacement, Cox-maze IV) were performed as needed (Table 1). Two cases required MVR, one with a Mosaic tissue valve (Medtronic, Inc) and one with an On-X mechanical valve (On-X Life Technologies, Austin, Tex). The reconstructed valves were tested for leaflet coaptation and competence, the left atrium was closed and the heart de-aired, and the patients were rewarmed and weaned from cardiopulmonary bypass. Valve function was confirmed using transesophageal echocardiography before chest closure.

\section{Echocardiographic Assessments}

All patients underwent preoperative and intraoperative transesophageal echocardiography. The patients were evaluated by transthoracic echocardiography approximately 1 week postoperatively, within 1 to 3 months postoperatively, and at least annually thereafter.

\section{RESULTS}

From September 2008 to February 2013, 19 patients underwent $\mathrm{MVr}$ or reconstruction requiring the addition of patch material. Detailed case information is listed in Table 1, and representative intraoperative photographs are shown in Figures 1 to 3. The etiology of MV dysfunction included calcific-degenerative disease in 6 , endocarditis in 5 , congenital disease in 3 , myxomatous disease in 2 , degeneration of previous repairs in 2 , and intraoperative correction of valve distortion secondary to aortic root replacement in 1 . The specific procedures included 12 leaflet reconstructions, 5 annular reconstructions after decalcification, and 2 combined leaflet reconstructions and annular reconstructions after decalcification. All were performed using the CorMatrix ECM product as the repair material.

The median duration of follow-up (calculated from the date of the procedure to the date of the latest echocardiographic evaluation) was 10.9 months (range, 4 days to 48 months). This included 8 patients for whom follow-up echocardiographic data $>12$ months were available.

Four of the MV procedures were second (redo) repairs and one was a third-time repair. Annuloplasty rings were used in 16 patients. Neochordae were implanted as a part of 7 repairs. Two annular decalcification cases required MVR. Concurrent procedures included tricuspid valve repair in 2, aortic valve replacement in 7 , repair of the ascending aorta and proximal arch in 1, Cox-maze IV in 6 , left atrial appendage occlusion in 1, coronary artery bypass grafting in 3 , and removal of an infected implantable cardioverter-defibrillator in 1 .

With 2 exceptions (detailed below), all patients demonstrated sustained repair integrity and valve competence throughout the follow-up period, with evidence of only mild leaflet thickening, zero to mild regurgitation, and no development of stenosis. No echocardiographic evidence of calcification of the ECM patch was identified in any patient. All patients experienced an improvement in New York Heart Association functional classification (Table 1).

\section{Adverse Events}

No early or late strokes occurred. The intraoperative and early postoperative complications were typical of complex valve operations in patients with severe comorbidities. They included new permanent pacemaker implantation in 4, blood transfusions in 7, methicillinresistant Staphylococcus aureus bacteremia in 1, and right ventricular failure requiring extracorporeal membrane 
TABLE 1. Relevant preoperative history and postoperative outcomes in a series of 19 patients undergoing mitral valve repair using CorMatrix ECM

\begin{tabular}{|c|c|c|c|c|c|c|c|c|c|c|}
\hline \multirow[b]{2}{*}{ Pt. No. } & \multirow[b]{2}{*}{ Age } & \multirow[b]{2}{*}{ Gender } & \multicolumn{3}{|c|}{ Preoperative scores } & \multirow{2}{*}{$\begin{array}{l}\text { MV disease etiology, } \\
\text { presentation, history, } \\
\text { and comorbidities }\end{array}$} & \multirow[b]{2}{*}{ Procedures* } & \multicolumn{3}{|c|}{$\begin{array}{l}\text { Postoperative echocardiographic } \\
\text { findings at longest follow-up visit }\end{array}$} \\
\hline & & & MR & NYHA & EuroSCORE & & & Outcomes & MR & NYHA \\
\hline 1 & 69 & $\mathrm{~F}$ & $4+$ & IV & 17 & $\begin{array}{l}\text { Posterior leaflet retraction } \\
\text { and fibrosis; } \\
\text { cardiogenic shock; } \\
\text { previous MVr (1997); } \\
\text { previous tracheostomy, } \\
\text { chemotherapy, and } \\
\text { radiotherapy for stage } \\
4 \text { laryngeal cancer; } \\
\text { severe pulmonary } \\
\text { HTN, AF, CRI, COPD, } \\
\text { HTN }\end{array}$ & $\begin{array}{l}\text { Redo MVr (posterior } \\
\text { leaflet ECM patch, P1- } \\
\text { P3); MV annuloplasty } \\
\text { ring; Cox-maze IV }\end{array}$ & $\begin{array}{l}\text { Normal valve function at } \\
3 \text { mo; endocarditis from } \\
\text { infected pacemaker } \\
\text { with severe MR at } 7 \\
\text { mo; reoperation with } \\
\text { bioprosthetic MVR; } \\
\text { incomplete healing of } \\
\text { patched area (see text } \\
\text { for details); late death } \\
\text { (pneumonia; } 2 \text { y) }\end{array}$ & 0 & NA \\
\hline 2 & 63 & M & $4+$ & IV & 28 & $\begin{array}{l}\text { Endocarditis with } \\
\text { dehiscence of previous } \\
\text { annuloplasty ring; } \\
\text { previous MVr (2007); } \\
\text { previous } \\
\text { esophagectomy, } \\
\text { chemotherapy, and } \\
\text { radiotherapy for } \\
\text { esophageal cancer; } \\
\text { CVA, DM, HTN }\end{array}$ & $\begin{array}{l}\text { Redo MVr (anterior leaflet } \\
\text { ECM patches to A1 and } \\
\text { A2-A3), MV } \\
\text { annuloplasty ring }\end{array}$ & $\begin{array}{l}\text { Normal valve function at } 6 \\
\text { mo; declining valvular } \\
\text { function ending in } \\
\text { reoperation at } 18 \text { mo } \\
\text { (MVR, AVR, TVr); } \\
\text { incomplete healing of } \\
\text { patched area (see text } \\
\text { for detail) }\end{array}$ & $1+$ & II \\
\hline 3 & 72 & $\mathrm{~F}$ & $4+$ & II & 2.5 & $\begin{array}{l}\text { Barlow valve bileaflet } \\
\text { involvement; bilateral } \\
\text { pleural effusion; } \\
\text { longstanding persistent } \\
\text { AF, HTN }\end{array}$ & $\begin{array}{l}\text { MV annular } \\
\text { decalcification and } \\
\text { ECM reconstruction; } 6 \\
\text { Gore-Tex neochordae; } \\
\text { MV annuloplasty ring; } \\
\text { TV annuloplasty ring; } \\
\text { Cox-Maze IV }\end{array}$ & $\begin{array}{l}\text { Early return to OR to } \\
\text { repair } \mathrm{AV} \text { tear caused } \\
\text { during } \mathrm{MVr} \text {; normal } \\
\text { valve function after } 4 \mathrm{y}\end{array}$ & $1+$ & I \\
\hline 4 & 68 & $\mathrm{~F}$ & $4+$ & IV & 29 & $\begin{array}{l}\text { Anterior leaflet } \\
\text { calcification and } \\
\text { annular dilatation; TR } \\
\text { (4+); bilateral pleural } \\
\text { effusion, severe } \\
\text { pulmonary HTN; } \\
\text { longstanding persistent } \\
\text { AF, ARI, CRI, DM, } \\
\text { HTN }\end{array}$ & $\begin{array}{l}\text { MVr (resection of } \\
\text { calcified anterior leaflet } \\
\text { and subtotal ECM patch } \\
\text { replacement; } \\
\text { commissural } \\
\text { decalcification); MV } \\
\text { annuloplasty ring, TV } \\
\text { annuloplasty ring; } \\
\text { Cox-maze IV }\end{array}$ & $\begin{array}{l}\text { Normal valve function at } \\
3 \mathrm{y} \text {; late death (ESRD; } \\
3.5 \mathrm{y})\end{array}$ & $1+$ & II \\
\hline 5 & 49 & $\mathrm{~F}$ & $4+$ & IV & 2.8 & $\begin{array}{l}\text { MV endocarditis, septic } \\
\text { shock, septic brain } \\
\text { emboli, multiple CVAs, } \\
\text { respiratory failure, DM, } \\
\text { HTN }\end{array}$ & $\begin{array}{l}\text { MVr (subtotal P1 leaflet } \\
\text { ECM patch } \\
\text { replacement); no } \\
\text { annuloplasty ring }\end{array}$ & $\begin{array}{l}\text { Normal valve function } \\
\text { at } 3.5 \mathrm{y}\end{array}$ & 0 & I \\
\hline 6 & 64 & $\mathrm{~F}$ & $2+$ & III & 20.5 & $\begin{array}{l}\text { Intraoperative distortion } \\
\text { of mitral annulus; } \\
\text { severe AS, moderate } \\
\text { AI; previous AVR } \\
\text { (2002), persistent AF, } \\
\text { HTN }\end{array}$ & $\begin{array}{l}\text { Redo aortic root } \\
\text { replacement, which } \\
\text { exacerbated MR; MVr } \\
\text { (anterior leaflet ECM } \\
\text { patch augmentation); } \\
\text { MV annuloplasty ring; } \\
\text { Cox-maze IV }\end{array}$ & $\begin{array}{l}\text { Normal valve function } \\
\text { at } 3 \mathrm{y}\end{array}$ & $1-2+$ & II \\
\hline 7 & 78 & $\mathrm{~F}$ & 4 & IV & 15 & $\begin{array}{l}\text { Healed endocarditis; CHF, } \\
\text { HTN }\end{array}$ & $\begin{array}{l}\mathrm{MVr} \text { (ECM patch repair of } \\
\text { large P2 defect; lateral } \\
\text { commissural chordal }\end{array}$ & $\begin{array}{l}\text { Normal valve function } \\
\text { at } 2.5 \mathrm{y}\end{array}$ & 0 & I \\
\hline
\end{tabular}




\begin{tabular}{|c|c|c|c|c|c|c|c|c|c|c|}
\hline \multirow[b]{2}{*}{ Pt. No. } & \multirow[b]{2}{*}{ Age } & \multirow[b]{2}{*}{ Gender } & \multicolumn{3}{|c|}{ Preoperative scores } & \multirow{2}{*}{$\begin{array}{l}\text { MV disease etiology, } \\
\text { presentation, history, } \\
\text { and comorbidities }\end{array}$} & \multirow[b]{2}{*}{ Procedures* } & \multicolumn{3}{|c|}{$\begin{array}{l}\text { Postoperative echocardiographic } \\
\text { findings at longest follow-up visit }\end{array}$} \\
\hline & & & MR & NYHA & EuroSCORE & & & Outcomes & MR & NYHA \\
\hline & & & & & & & $\begin{array}{l}\text { and papillary } \\
\text { fenestration); MV } \\
\text { annuloplasty ring }\end{array}$ & & & \\
\hline 8 & 58 & M & $4+$ & IV & 2.3 & $\begin{array}{l}\text { Bileaflet myxomatous } \\
\text { disease and extensive } \\
\text { annular calcification; } \\
\text { HTN }\end{array}$ & $\begin{array}{l}\text { MV annular } \\
\text { decalcification and } \\
\text { ECM reconstruction; } \\
\text { MVr (posterior leaflet } \\
\text { sliding plasty); } 8 \text { Gore- } \\
\text { Tex neochordae; MV } \\
\text { annuloplasty ring; LAA } \\
\text { clip }\end{array}$ & $\begin{array}{l}\text { Normal valve function } \\
\text { at } 2.5 \mathrm{y}\end{array}$ & 0 & I \\
\hline 9 & 29 & $\mathrm{~F}$ & $4+$ & III & 5.5 & $\begin{array}{l}\text { Cleft anterior leaflet and } \\
\text { inadequate posterior } \\
\text { leaflet surface area; } 2 \\
\text { previous } \mathrm{MVr} \text { (aged } 2 \\
\text { and } 8 \mathrm{y} \text { ); persistent } \mathrm{AF}\end{array}$ & $\begin{array}{l}\text { Redo-redo MVr (ECM } \\
\text { patch augmentation of } \\
\text { P2 and P3); } 4 \text { Gore-Tex } \\
\text { neochordae; MV } \\
\text { annuloplasty ring; Cox } \\
\text { maze IV }\end{array}$ & $\begin{array}{l}\text { Normal valve function } \\
\text { at } 1.75 \mathrm{y}\end{array}$ & 0 & I \\
\hline 10 & 60 & $\mathrm{~F}$ & $4+$ & III & 7 & $\begin{array}{l}\text { Calcific restriction of P3; } \\
\text { 3+ AI, aneurysm of } \\
\text { ascending aorta; } \\
\text { persistent AF, HTN, } \\
\text { Turner syndrome }\end{array}$ & $\begin{array}{l}\text { AVR; ascending aorta and } \\
\text { proximal arch repair; } \\
\text { MV annular } \\
\text { decalcification and } \\
\text { ECM reconstruction; } \\
\text { MVr (P3 ECM patch } \\
\text { augmentation); } 4 \text { Gore- } \\
\text { Tex neochordae; MV } \\
\text { annuloplasty ring }\end{array}$ & $\begin{array}{l}\text { Normal valve function } \\
\text { at } 1.25 \mathrm{y}\end{array}$ & $1+$ & I-II \\
\hline 11 & 65 & M & $1+$ & IV & 26.5 & $\begin{array}{l}\text { Severe calcific MS and } \\
\text { AS; multivessel CAD } \\
\text { with left main stenosis, } \\
\text { near-circumferential } \\
\text { MV annular } \\
\text { calcification with } \\
\text { ventricular extension; } \\
\text { bilateral pleural } \\
\text { effusion, severe } \\
\text { pulmonary HTN; } \\
\text { previous renal } \\
\text { transplant (1998); } \\
\text { severe COPD, DM, } \\
\text { HTN }\end{array}$ & $\begin{array}{l}\text { MV annular } \\
\text { decalcification and } \\
\text { ECM reconstruction; } \\
\text { MVR and AVR; } \\
\text { CABG; ECMO }\end{array}$ & $\begin{array}{l}\text { Repair and prosthetic } \\
\text { valves intact with good } \\
\text { biventricular function } \\
\text { at } 4 \mathrm{~d} \text {; early death } \\
\text { (MSOF; } 7 \mathrm{~d} \text { ) }\end{array}$ & NA & NA \\
\hline 12 & 71 & M & $4+$ & III & 50.3 & $\begin{array}{c}\text { Calcific MS; recent MI; } \\
\text { severe AS; previous } \\
\text { CABG (1996); CAD, } \\
\text { COPD, DM, HTN }\end{array}$ & $\begin{array}{l}\text { MV annular } \\
\text { decalcification and } \\
\text { ECM reconstruction; } \\
\text { MVR and AVR; CABG }\end{array}$ & $\begin{array}{l}\text { Normal prosthetic valve } \\
\text { function at } 11 \mathrm{mo}\end{array}$ & 0 & I \\
\hline 13 & 69 & $\mathrm{~F}$ & $4+$ & III & 5.1 & $\begin{array}{l}\text { Severe MR, moderate MS } \\
\text { after previous MVr } \\
\text { (2007); COPD, HTN, } \\
\text { RA }\end{array}$ & $\begin{array}{l}\text { Redo } \mathrm{MVr} \text { (ECM patch } \\
\text { augmentation of P2 and } \\
\mathrm{P} 3 \text { ); MV annuloplasty } \\
\text { ring }\end{array}$ & $\begin{array}{l}\text { Normal valve function } \\
\text { at } 11.5 \mathrm{mo}\end{array}$ & $1+$ & I-II \\
\hline 14 & 37 & $\mathrm{~F}$ & $4+$ & II & 0.8 & $\begin{array}{l}\text { Congenitally aberrant P3; } \\
\text { isolated severe MR }\end{array}$ & $\begin{array}{l}\mathrm{MVr} \text { (ECM patch } \\
\text { augmentation of P2 and } \\
\text { P3 leaflets); } 6 \text { Gore-Tex } \\
\text { neochordae; MV } \\
\text { annuloplasty ring } \\
\end{array}$ & $\begin{array}{l}\text { Normal valve function } \\
\text { at } 7.5 \text { mo }\end{array}$ & 0 & I \\
\hline
\end{tabular}

\section{disease and extensive} annular calcification HTN

eft anterior leaflet and dequate posterior previous $\mathrm{MVr}$ (aged 2

lcific restriction of $\mathrm{P} 3$ persistent AF, HTN Turner syndrome

vere calcific MS and near-circumferential ventricular extension; previous renal severe COPD, DM, severe AS; previous CABG (1996); CAD, after previous $\mathrm{MVr}$ (2007); COPD, HTN,

ongenitally aberrant $\mathrm{P} 3$, annuloplasty ring 


\begin{tabular}{|c|c|c|c|c|c|c|c|c|c|c|}
\hline \multirow[b]{2}{*}{ Pt. No. } & \multirow[b]{2}{*}{ Age } & \multirow[b]{2}{*}{ Gender } & \multicolumn{3}{|c|}{ Preoperative scores } & \multirow{2}{*}{$\begin{array}{l}\text { MV disease etiology, } \\
\text { presentation, history, } \\
\text { and comorbidities }\end{array}$} & \multirow[b]{2}{*}{ Procedures* } & \multicolumn{3}{|c|}{$\begin{array}{l}\text { Postoperative echocardiographic } \\
\text { findings at longest follow-up visit }\end{array}$} \\
\hline & & & MR & NYHA & EuroSCORE & & & Outcomes & MR & NYHA \\
\hline 15 & 61 & M & $4+$ & III & 1.6 & $\begin{array}{l}\text { Calcific restriction of P1 } \\
\text { and P2; moderate AS; } \\
\text { bicuspid AV; HTN }\end{array}$ & $\begin{array}{l}\text { MV annular } \\
\text { decalcification and } \\
\text { ECM reconstruction; } \\
\text { MVr (ECM patch } \\
\text { augmentation of P1 and } \\
\text { P2); MV annuloplasty } \\
\text { ring; AVR }\end{array}$ & $\begin{array}{l}\text { Normal valve function } \\
\text { at } 1.5 \mathrm{mo}\end{array}$ & 0 & I \\
\hline 16 & 73 & M & $4+$ & III & 5.6 & MV endocarditis; ARF & $\begin{array}{l}\text { MVr (debridement and } \\
\text { ECM patch } \\
\text { reconstruction of P2); } 4 \\
\text { Gore-Tex neochordae; } \\
\text { MV annuloplasty ring; } \\
\text { CABG }\end{array}$ & $\begin{array}{l}\text { Normal valve function } \\
\text { at } 1.5 \mathrm{mo}\end{array}$ & 0 & I \\
\hline 17 & 67 & M & $4+$ & III & 4.2 & $\begin{array}{l}\mathrm{P} 1 \text { and } \mathrm{P} 2 \text { restriction } \\
\text { status after previous } \\
\mathrm{MVr}(2007) ; \text { persistent } \\
\mathrm{AF}\end{array}$ & $\begin{array}{l}\text { Redo MVr (ECM patch } \\
\text { augmentation of P1 and } \\
\text { P2); MV annuloplasty } \\
\text { ring; Cox-maze IV }\end{array}$ & $\begin{array}{l}\text { Normal valve function } \\
\text { at } 1.5 \mathrm{mo}\end{array}$ & 0 & I \\
\hline 18 & 44 & M & $4+$ & IV & 3 & $\begin{array}{l}\text { Bileaflet myxomatous } \\
\text { MR; extensive annular } \\
\text { calcification; } 1+\mathrm{TR} \\
\text { with annular dilation; } \\
\text { pulmonary edema; } \\
\text { severe pulmonary HTN }\end{array}$ & $\begin{array}{l}\text { MV annular } \\
\text { decalcification and } \\
\text { ECM reconstruction; } \\
\text { MVr (posterior leaflet } \\
\text { sliding plasty); chordal } \\
\text { transfer and 6 Gore-Tex } \\
\text { neochordae; MV } \\
\text { annuloplasty ring; TV } \\
\text { annuloplasty ring; LAA } \\
\text { clip }\end{array}$ & $\begin{array}{l}\text { Normal valve function } \\
\text { at } 1.5 \mathrm{mo}\end{array}$ & 0 & I \\
\hline 19 & 35 & M & $3+$ & III & 1.9 & $\begin{array}{l}\text { AV-MV endocarditis with } \\
\text { 3-4+ AI and MV } \\
\text { perforation; splenic } \\
\text { venous thrombosis, } \\
\text { positive lupus } \\
\text { anticoagulant, HTN }\end{array}$ & $\begin{array}{l}\text { MVr (anterior leaflet } \\
\text { debridement and ECM } \\
\text { patch repair); MV } \\
\text { annuloplasty ring; AVR }\end{array}$ & $\begin{array}{l}\text { Normal valve function } \\
\text { at } 1.3 \mathrm{mo}\end{array}$ & 0 & I \\
\hline
\end{tabular}

$\overline{E C M}$, CorMatrix extracellular matrix bioscaffold; Pt. No., patient number; $M R$, mitral regurgitation; NYHA, New York Heart Association (functional classification); EuroSCORE, EuroSCORE operative mortality risk; $M V$, mitral valve; $F$, female; $M V r$, mitral valve repair; $H T N$, hypertension; $A F$, atrial fibrillation; $C R I$, chronic renal insufficiency; $C O P D$, chronic pulmonary obstructive disease; $M R$, mitral regurgitation; $M V R$, mitral valve replacement; $N A$, not available; $M$, male; $C V A$, cerebrovascular event; $D M$, diabetes mellitus; $A V R$, aortic valve replacement; $T V r$, tricuspid valve repair; $T V$, tricuspid valve; $O R$, operating room; $A V$, aortic valve; $T R$, tricuspid regurgitation; $A R I$, acute renal insufficiency; $E S R D$, end-stage renal disease; $C V A$, cerebrovascular accident; $A S$, aortic stenosis; $A I$, aortic insufficiency; $C H F$, congestive heart failure; $L A A$, left atrial appendage; $C A D$, coronary artery disease; $C A B G$, coronary artery bypass grafting; $E C M O$, extracorporeal membrane oxygenation; $M S O F$, multisystem organ failure; $M S$, mitral stenosis; $M I$, myocardial infarction; $R A$, rheumatoid arthritis; $A R F$, acute renal failure. *See "Methods" section for prosthetic valve and annuloplasty ring types.

oxygenation in 1 . One patient was returned to the operating room because of new-onset aortic insufficiency attributable to inadvertent damage during MVr. The tear in the aortic valve leaflet was repaired with a small patch of CorMatrix ECM bioscaffold. Systolic anterior motion was not detected in any patient.

One early death occurred on the seventh postoperative day from multisystem organ failure after combined MVR, aortic valve replacement, and coronary artery bypass grafting. This patient had presented in cardiogenic shock with a history of severe pulmonary hypertension and previous renal transplantation (EuroSCORE, 26.5).
Two late deaths occurred in the present series. Patient 4 died 3 years, 7 months postoperatively of end-stage renal disease. Patient 1, who had a history significant for laryngeal cancer, chemotherapy, radiotherapy, tracheostomy, and recurrent pneumonia, had undergone redo $\mathrm{MVr}$ with an ECM bioscaffold patch of the posterior leaflet. She presented 7 months postoperatively with endocarditis originating from her pacemaker and severe recurrent mitral regurgitation. On reoperation, 1 of the leaflet sutures was found to be broken. The ECM patch did not appear to have healed or undergone reconstructive remodeling, and a segment at the edge of the patch appeared to have torn 


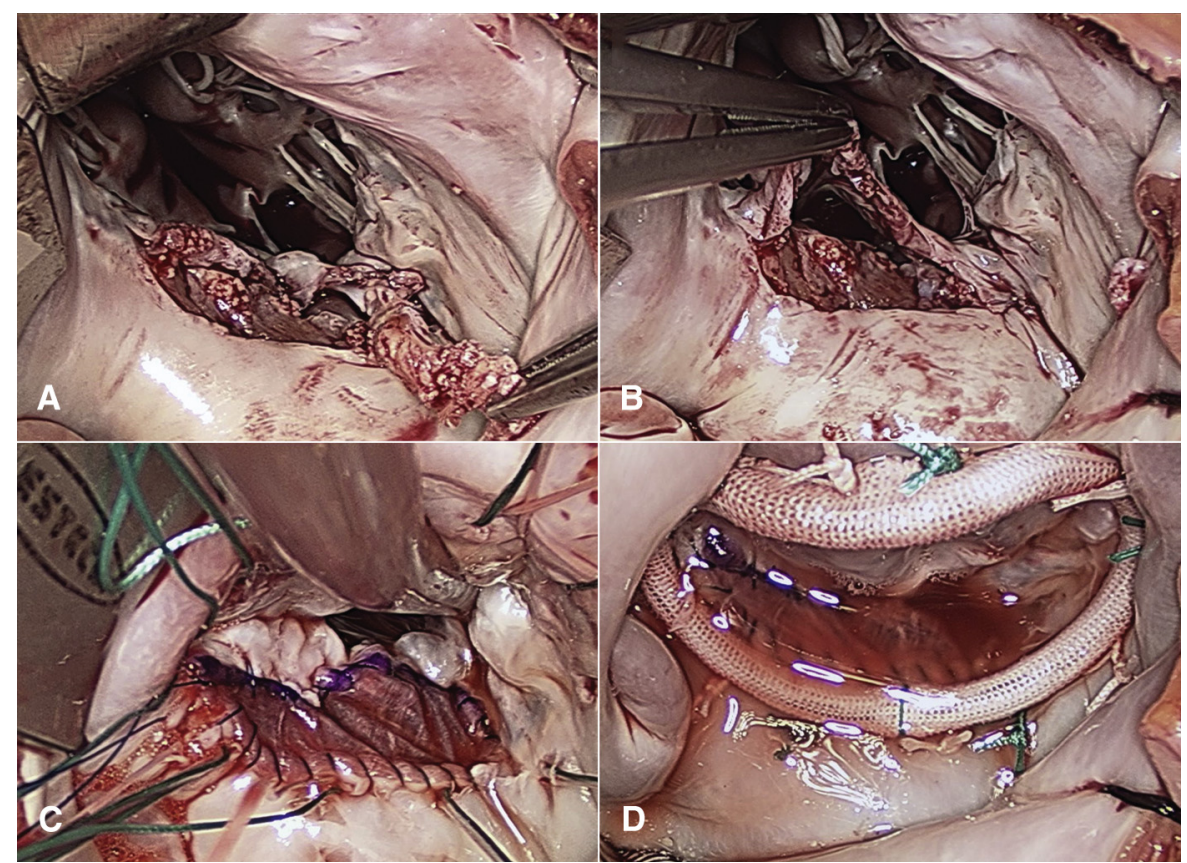

FIGURE 1. Patient 15, posterior leaflet repair. A and B, Decalcification of the mitral valve annulus and P1-P2 segments. C, Extracellular matrix bioscaffold patch augmentation. D, Completed mitral valve repair tested under pressure for valve competence.

away from the suture line. The patient recovered after receiving a bioprosthetic MV and repair of the tricuspid valve and subsequently returned to work. However, she died of pneumonia 15 months after the second operation.

A second occurrence of incomplete graft integration developed in 1 patient who remained alive. This patient's history was also significant for cancer (esophageal), chemotherapy, radiotherapy, and esophagectomy. He had originally presented with endocarditis and ring dehiscence of a previous MVr. He recovered well after redo patch repairs to the $\mathrm{A} 1$ and $\mathrm{A} 2-\mathrm{A} 3$ portions of the anterior leaflet. However, 1 year after surgery, evidence was found of

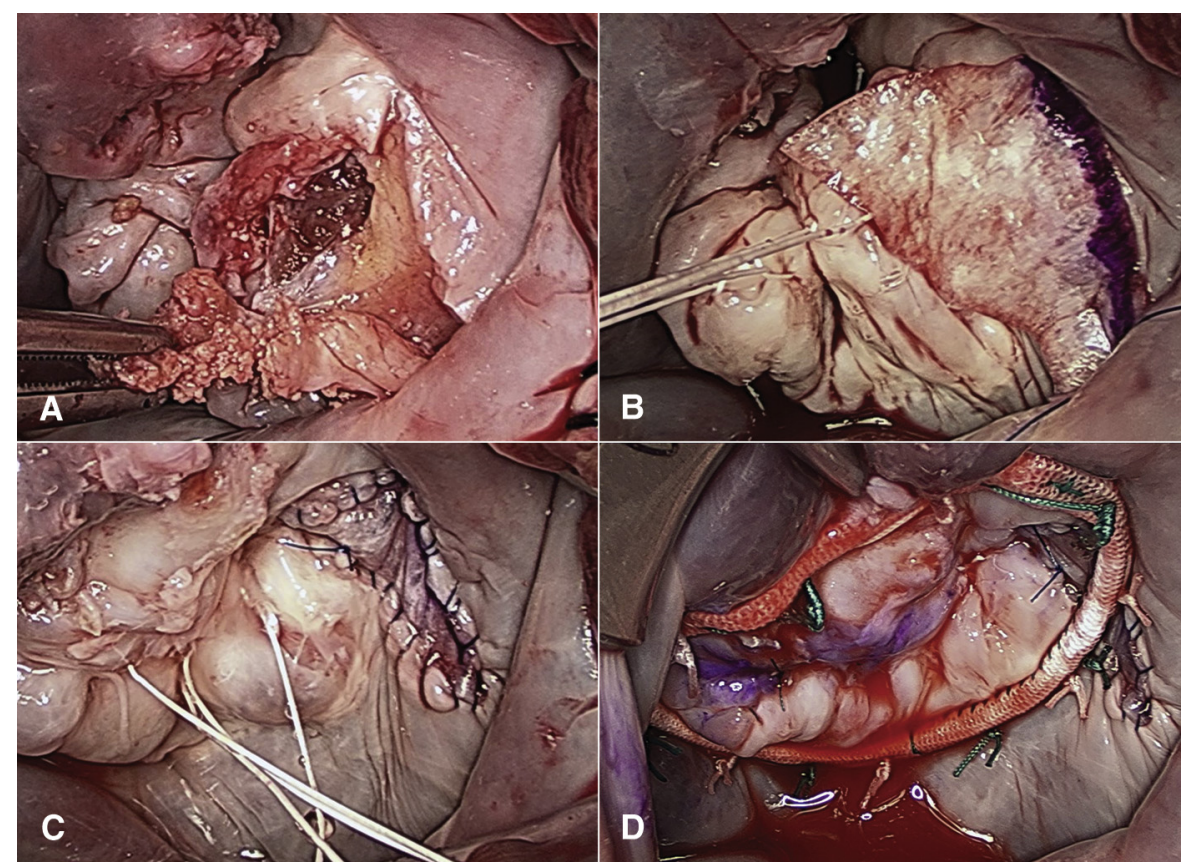

FIGURE 2. Patient 10, decalcification and repair of annulus and P3. A, Debridement of calcified tissue. B, Extracellular matrix bioscaffold patch augmentation. C, Adjustment of neochordae. D, Completed mitral valve repair. 


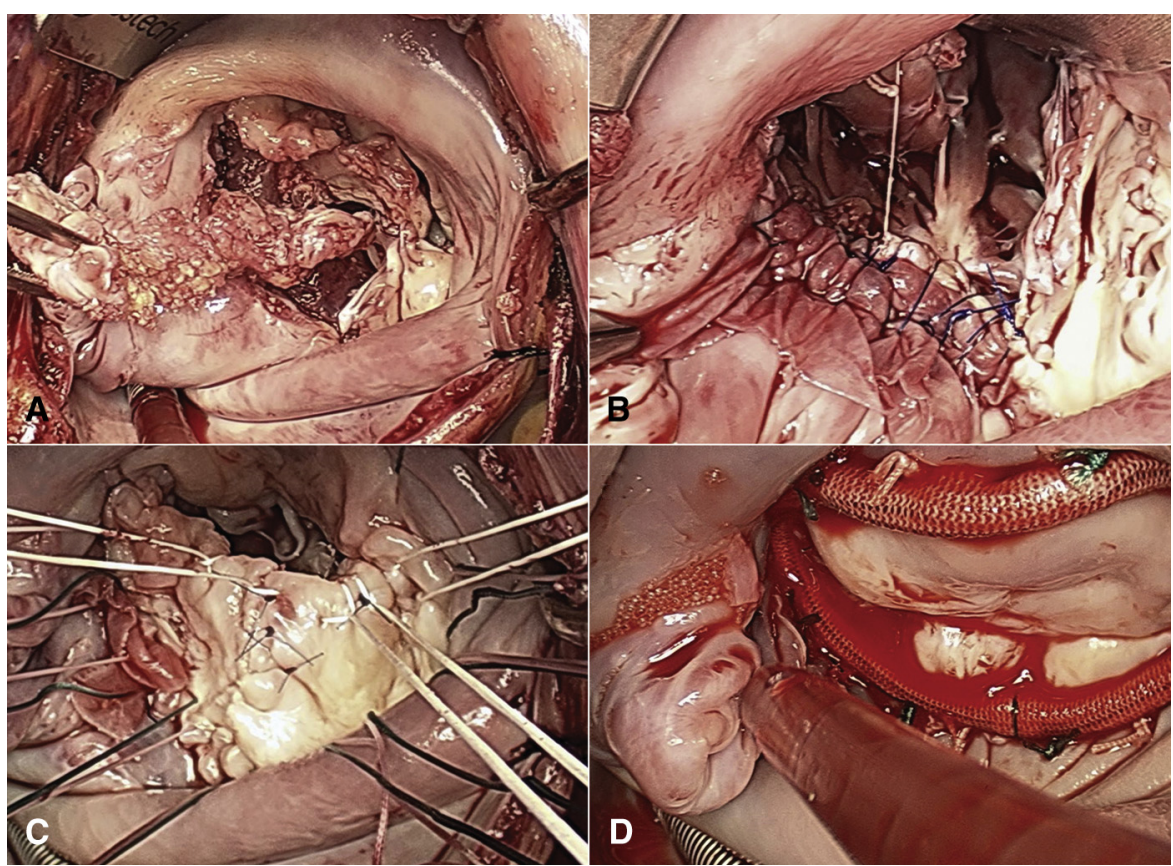

FIGURE 3. Patient 18, annular decalcification and leaflet repair. A, Decalcification. B, Annular reconstruction with extracellular matrix bioscaffold. $\mathrm{C}$, Leaflet reattached and neochordae implanted. D, Completed mitral valve repair.

progressive asymptomatic MV regurgitation. He became symptomatic 18 months postoperatively and underwent reoperation. Visual inspection suggested that although the ECM patch repair to A1 had healed completely, healing of the A2-A3 patch was incomplete along the edge adjoining the A3 segment. Histopathologic examination showed that portions of the patch distal to the suture line contained CD31-positive staining consistent with vascularization, and the sections close to the proximal edge appeared avascular (Figure 4). Because the patient had also developed grade $3+$ aortic and tricuspid insufficiency, his mitral and aortic valves were replaced, and his tricuspid valve was repaired. He recovered well and was still alive at the latest follow-up visit.

\section{DISCUSSION}

To our knowledge, the present study reports the longest follow-up data on the clinical use of the ECM bioscaffold for MVr. Consistent with findings from preclinical studies, published clinical cases, and small series, ${ }^{23-30}$ the results of our clinical experience suggest that human cardiac valve repairs using the ECM bioscaffold remain structurally strong and durable at midterm follow-up. No evidence was found of an acute or a chronic inflammatory reaction, and the ECM bioscaffold was pliable and easily tailored for applications commonly encountered during valve surgery. Our patient cohort experienced no strokes, an issue of primary concern for intracardiac foreign body implantation. Calcification was never identified in the

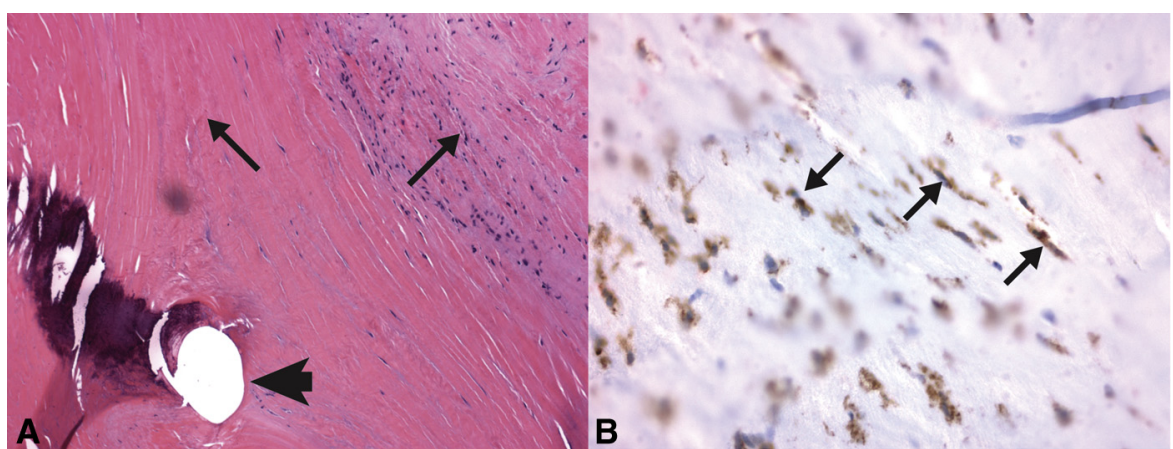

FIGURE 4. Patient 2, histopathologic results from explantation from a patient with a history of chemotherapy and radiotherapy. A, Hematoxylin-eosin stain $(\times 100)$ showing regions of vascularized (right arrow) and avascular (left arrow) graft material. The arrowhead denotes the hole left by the suture. B, Immunohistochemical stain for CD31 $(\times 400)$. Positive areas indicate probable endothelial cells, suggesting partial vascularization of some regions of the explanted graft (arrows). 
ECM bioscaffold patches on echocardiography. This included our patient with end-stage renal disease at implantation, who had died 3 years, 7 months after surgery, and both of the patients who experienced patch failure. Using qualitative echocardiographic assessment, the ECM bioscaffold geometry appeared grossly preserved in all patients without visually obvious contraction or elongation of the leaflet patches.

Our experience has provided an opportunity to monitor the performance of the ECM bioscaffold in 3 patients with active endocarditis (patients 2, 5, and 16). Consistent with the experience of Sundermann and colleagues, ${ }^{28}$ no recurrences of infection have developed $\leq 3.5$ years after surgery.

In another case (patient 6), an aortic annular enlargement and replacement of the aortic root resulted in unanticipated distortion of the MV architecture such that the leaflets were no longer capable of coaptation. Situations in which the primary intervention leads to anatomic changes elsewhere in the heart can be challenging to resolve. In patient 6 , the ECM bioscaffold was used to perform MV leaflet augmentation to restore coaptation and valve function.

The ECM bioscaffold did not appear to integrate completely in patients 1 and 2 (Figure 4). These patients had in common a history of high-dose chemotherapy and radiotherapy for cancer. Although the cause of graft failure was not definitively determined, it is plausible that the cancer treatment negatively affected the patients' resident population of pluripotent cells. Because the suggested mechanism behind constructive ECM remodeling is native stem cell infiltration, proliferation, and differentiation, ${ }^{12}$ it is possible that the previous chemotherapy and/or radiotherapy interfered with the patients' intrinsic ability to repopulate the ECM bioscaffold. These cases are anecdotal, and additional experience is necessary to determine the relationship between previous chemotherapy or radiotherapy and successful ECM bioscaffold implantation and integration. Until more definitive answers are available, caution is advised when using the ECM bioscaffold in patients who have undergone treatments that substantially affect the number and function of stem or progenitor cells.

The present report was subject to the limitations of a small retrospective case series. Although some patients have been followed for several years, the median followup period of 10.9 months does not allow any definitive conclusions about the long-term durability of the repairs. No opportunities were available for direct or microscopic observation of the ECM bioscaffold in healthy patients after an extended period of implantation. Also, no opportunity was available to make direct comparisons with autologous pericardium or other surgical materials used for this application. Although 1 of the postulated benefits of the ECM material is that it might be capable of accommodating somatic growth, ${ }^{31}$ the limited nature of our adult population did not allow an evaluation of this property. Other groups are currently using this material in pediatric populations, and any findings with regard to somatic growth potential would be of great interest. ${ }^{24-26}$

\section{CONCLUSIONS}

The present results suggest the ECM bioscaffold could be a convenient and safe option for $\mathrm{MVr}$ and for support of prosthetic valve implantation after annular decalcification in adults, with caution urged regarding patients who have previously undergone radiotherapy and/or chemotherapy. Continued follow-up and additional studies are needed to appreciate fully the ECM bioscaffold's resistance to long-term inflammation and calcification in humans, its remodeling dynamics in unusual patient populations, and its utility compared with other repair materials.

We thank Jennifer Lockliear, PAC, for her contributions and assistance with data collection. Jeanne McAdara-Berkowitz, $\mathrm{PhD}$, provided professional editing assistance, funded by CorMatrix Cardiovascular, Inc.

\section{References}

1. Verbrugghe P, Meuris B, Flameng W, Herijgers P. Reconstruction of atrioventricular valves with photo-oxidized bovine pericardium. Interact Cardiovasc Thorac Surg. 2009;9:775-9.

2. Li X, Guo Y, Ziegler KR, Model LS, Eghbalieh SD, Brenes RA, et al. Current usage and future directions for the bovine pericardial patch. Ann Vasc Surg. 2011;25:561-8.

3. Sakuma K, Iguchi A, Ikada Y, Tabayashi K. Closure of the pericardium using synthetic bioabsorbable polymers. Ann Thorac Surg. 2005;80:1835-40.

4. Us MH, Sungun M, Sanioglu S, Pocan S, Cebeci BS, Ogus T, et al. A retrospective comparison of bovine pericardium and polytetrafluoroethylene patch for closure of ventricular septal defects. J Int Med Res. 2004;32:218-21.

5. Ozeren M, Han U, Mavioglu I, Simsek E, Soyal MF, Guler G, et al. Consequences of PTFE membrane used for prevention of re-entry injuries in rheumatic valve disease. Cardiovasc Surg. 2002;10:489-93.

6. Umashankar PR, Mohanan PV, Kumari TV. Glutaraldehyde treatment elicits toxic response compared to decellularization in bovine pericardium. Toxicol Int. 2012;19:51-8.

7. Sinha P, Zurakowski D, Kumar TK, He D, Rossi C, Jonas RA. Effects of glutaraldehyde concentration, pretreatment time, and type of tissue (porcine versus bovine) on postimplantation calcification. J Thorac Cardiovasc Surg. 2012;143:224-7.

8. Dillon J, Yakub MA, Nordin MN, Pau KK, Krishna Moorthy PS. Leaflet extension in rheumatic mitral valve reconstruction. Eur J Cardiothorac Surg. 2013;44:682-9.

9. Aubert S, Flecher E, Rubin S, Acar C, Gandjbakhch I. Anterior mitral leaflet augmentation with autologous pericardium. Ann Thorac Surg. 2007;83: 1560- 1

10. Ng CK, Nesser J, Punzengruber C, Pachinger O, Auer J, Franke H, et al Valvuloplasty with glutaraldehyde-treated autologous pericardium in patients with complex mitral valve pathology. Ann Thorac Surg. 2001;71:78-85.

11. Chauvaud S, Jebara V, Chachques JC, el Asmar B, Mihaileanu S, Perier P, et al Valve extension with glutaraldehyde-preserved autologous pericardium: results in mitral valve repair. J Thorac Cardiovasc Surg. 1991;102:171-7; discussion 177.

12. Badylak SF, Freytes DO, Gilbert TW. Extracellular matrix as a biologica scaffold material: structure and function. Acta Biomater. 2009;5:1-13.

13. Badylak S, Obermiller J, Geddes L, Matheny R. Extracellular matrix for myocardial repair. Heart Surg Forum. 2003;6:E20-6.

14. Badylak SF. Xenogeneic extracellular matrix as a scaffold for tissue reconstruction. Transpl Immunol. 2004;12:367-77. 
15. Robinson KA, Li J, Mathison M, Redkar A, Cui J, Chronos NA, et al. Extracellular matrix scaffold for cardiac repair. Circulation. 2005;112: I135-43.

16. Zhao ZQ, Puskas JD, Xu D, Wang NP, Mosunjac M, Guyton RA, et al. Improvement in cardiac function with small intestine extracellular matrix is associated with recruitment of C-kit cells, myofibroblasts, and macrophages after myocardial infarction. J Am Coll Cardiol. 2010;55: 1250-61.

17. Zantop T, Gilbert TW, Yoder MC, Badylak SF. Extracellular matrix scaffolds are repopulated by bone marrow-derived cells in a mouse model of Achilles tendon reconstruction. J Orthop Res. 2006;24:1299-309.

18. Badylak SF, Gilbert TW. Immune response to biologic scaffold materials. Semin Immunol. 2008;20:109-16.

19. Daly KA, Stewart-Akers AM, Hara H, Ezzelarab M, Long C, Cordero K, et al. Effect of the alphaGal epitope on the response to small intestinal submucosa extracellular matrix in a nonhuman primate model. Tissue Eng Part A. 2009; 15:3877-88.

20. Mascagni R, Al Attar N, Lamarra M, Calvi S, Tripodi A, Mebazaa A, et al. Edge-to-edge technique to treat post-mitral valve repair systolic anterior motion and left ventricular outflow tract obstruction. Ann Thorac Surg. 2005;79:471-3; discussion 474.

21. Quigley RL, Garcia FC, Badawi RA. Prevention of systolic anterior motion after mitral valve repair with an anterior leaflet valvuloplasty. J Heart Valve Dis. 2004; 13:927-30.

22. Perier P, Clausnizer B, Mistarz K. Carpentier "sliding leaflet" technique for repair of the mitral valve: early results. Ann Thorac Surg. 1994;57: $383-6$.
23. Gerdisch MW, Akinwande AO, Matheny RG. Use of a novel acellular xenograft as a patch for aortic annular enlargement during aortic valve replacement. Innovations. 2010;5:60-2.

24. Quarti A, Nardone S, Colaneri M, Santoro G, Pozzi M. Preliminary experience in the use of an extracellular matrix to repair congenital heart diseases. Interact Cardiovasc Thorac Surg. 2011;13:569-72.

25. Quarti A, Nardone S, Colaneri M, Santoro G, Pozzi M. Valve leaflet reconstruction in pediatrics with extracellular matrix patch. J Heart Valve Dis. 2012;21:138-9.

26. Scholl FG, Boucek MM, Chan KC, Valdes-Cruz L, Perryman R. Preliminary experience with cardiac reconstruction using decellularized porcine extracellular matrix scaffold: human applications in congenital heart disease. World J Pediatr Congenit Heart Surg. 2010;1:132-6.

27. Smith CR, Stamou SC, Boeve TJ, Hooker RC. Repair of a penetrating ascending aortic ulcer with localized resection and extracellular matrix patch aortoplasty. Ann Thorac Surg. 2012;94:988-9.

28. Sundermann SH, Biefer HR, Emmert MY, Falk V. Use of extracellular matrix materials in patients with endocarditis. Thorac Cardiovasc Surg. November 9, 2012 [Epub ahead of print].

29. Witt RG, Raff G, Van Gundy J, Rodgers-Ohlau M, Si MS. Short-term experience of porcine small intestinal submucosa patches in paediatric cardiovascular surgery. Eur J Cardiothorac Surg. 2013;44:72-6.

30. Wyler von Ballmoos M, Murtaza G, Gasparri M, Masroor S. Reconstruction of the right atrium using an extracellular matrix patch in a patient with severe mediastinal aspergillosis. Int J Surg Case Rep. 2013;4:290-2.

31. Robotin-Johnson MC, Swanson PE, Johnson DC, Schuessler RB, Cox JL An experimental model of small intestinal submucosa as a growing vascular graft. J Thorac Cardiovasc Surg. 1998;116:805-11. 